\title{
Peripherally Presented Emotional Scenes: A Spatiotemporal Analysis of Early ERP Responses
}

\author{
Simon Rigoulot $\cdot$ Sylvain Delplanque $\cdot$ Pascal Despretz $\cdot$ Sabine Defoort-Dhellemmes \\ Jacques Honoré $\cdot$ Henrique Sequeira
}

Accepted: 14 February 2008/Published online: 12 March 2008

(C) Springer Science+Business Media, LLC 2008

\begin{abstract}
Recent findings from event-related potentials (ERPs) studies provided strong evidence that centrally presented emotional pictures could be used to assess affective processing. Moreover, several studies showed that emotionally charged stimuli may automatically attract attention even if these are not consciously identified. Indeed, such perceptive conditions can be compared to those typical of the peripheral vision, particularly known to have low spatial resolution capacities. The aim of the present study was to characterize at behavioral and neural levels the impact of emotional visual scenes presented in peripheral vision. Eighteen participants were asked to categorize neutral and unpleasant pictures presented at central $\left(0^{\circ}\right)$ and peripheral eccentricities $\left(-30\right.$ and $\left.+30^{\circ}\right)$ while ERPs were recorded from 63 electrodes. ERPs were analysed by means of spatio-temporal principal component analyses (PCA) in order to evaluate influences of the emotional content on ERP components for each spatial position (central vs. peripheral). Main results highlight that affective modulation of early ERP components exists for both centrally and peripherally presented pictures. These
\end{abstract}

S. Rigoulot $(\bowtie) \cdot$ P. Despretz $\cdot$ S. Defoort-Dhellemmes .

J. Honoré · H. Sequeira

Neurosciences Fonctionnelles \& Pathologies, CNRS,

UMR 8160, Service EFV, CHRU-Université de Lille II,

59037 Lille, France

e-mail: s-rigoulot@chru-lille.fr

S. Delplanque

Swiss Center for Affective Sciences, CISA, University of

Geneva, 7, rue des Battoirs, 1205 Geneva, Switzerland

H. Sequeira

Neurosciences, SN4.1, Université de Lille I,

59655 Villeneuve d'Ascq, France findings suggest that, for far peripheral eccentricities as for central vision, the brain engages specific resources to process emotional information.

Keywords Emotion - Peripheral vision - ERP . Spatial frequency $\cdot$ Spatio-temporal PCA

\section{Introduction}

The visual detection of natural emotional scenes constitutes an essential adaptive ability. However, human visual capacities are not homogeneous depending on which part of retina is stimulated. Indeed, the degradation of visual performance with retinal eccentricity strongly decreases the capability to apprehend several kinds of environmental stimuli (e.g., [1]). Thus, the detection of one stimulus in our surrounding world, mainly projected on peripheral retina, causes saccades which displace targets of interest into foveal vision where detailed visual analysis can be performed [2]. These differential visual aptitudes beginning in the retina are subtended by the two major and parallel retino-geniculo-cortical pathways, the parvocellular and the magnocellular systems. Contrary to the parvocellular system which takes its origin mainly from central part of the retina [3] and is particularly sensitive to contrast, high spatial frequency and colour, the magnocellular system begins essentially in peripheral parts of the retina, is less effective in contrast detection and is not responsive to colour. However, the magnocellular pathway possesses a high temporal resolution $[4,5]$.

Several studies evidenced the low visual acuity in peripheral vision with letters [6], digits [7] or psychophysical stimuli such as sinusoidal gratings [8] or compound Gabor patterns [9]. However, using ecological 
or complex stimuli such as objects or natural scenes [10, 11], some studies showed unexpected good performances in objects recognition (Jebara et al., submitted), in colour detection [12] or in a categorization task [13]. In particular, Thorpe and colleagues interpreted the high performance to the categorization of animal pictures as a phylogenetic advantage for species adaptation and individual survival. In sum, this kind of results evoked the possibility that the peripheral vision could process stimuli having a very high adaptive value.

In an evolutionary perspective, the particular value of emotions is unquestionable and much specificity associated with emotional processing has been demonstrated through behavioural and electrophysiological studies. Indeed, using experimental material presented in central vision, it has been demonstrated that emotional stimuli, especially unpleasant ones, are preferentially processed in comparison with neutral stimuli $[14,15]$. Moreover, emotional stimuli are better remembered [16-18], and can modulate early [19] and late [20] evoked components.

The privileged attentional capture of emotional stimuli can persist under spatio-temporal constraints very similar to those of peripheral vision, as showed by techniques of visual masking [21], very fast presentations [22, 23] and binocular rivalry [24]. Indeed, Calvo and Lang [25] explored parafoveal vision at $10^{\circ}$ of eccentricity and evidenced a preferential gaze attraction for emotional pictures when compared with neutral ones. At the same eccentricity, they also showed that emotional pictures were better remembered, even with brief presentations (150 ms).

Taken together, these data allow hypothesizing that, despite the low acuity of the peripheral vision, emotional processing exists when stimuli are presented to high eccentricity. Consequently, the aim of the current study was (1) to characterize the early visual processing in response to pictures presented at high eccentricities and (2) to evidence the existence of early emotional processing at those eccentricities. To this end, visual unpleasant and neutral scenes selected from the International Affective Picture System (IAPS; [26]) were controlled with regards to their physical parameters $[25,27]$ and were presented in central and peripheral vision.

The behavioral and electrophysiological impact of such stimulations was evaluated by means of reaction times and event-related potentials (ERPs) recordings. Moreover, considering the rare available electrophysiological data related to peripheral vision, an exploratory spatio-temporal principal component analysis (PCA) was chosen to investigate the ERPs data (see [28]). Indeed, PCA techniques have already demonstrated their relevance to explore ERPs in response to emotional stimuli (e.g., [20, 29-32]).

\section{Methods}

Eighteen right-handed healthy undergraduate female students (mean $20.5 \pm 2.2$ years) were included in the study. They all had normal or corrected to normal vision. Prior to the experiment, participants were given questionnaires in order to test their handedness [33] and to verify that they had no history of neurological or psychiatric disorder, or drug consumption.

One hundred and thirty-eight pictures were selected from IAPS ${ }^{1}[26]$ and from a dataset developed in our laboratory (38 pictures rated for their emotional valence and arousal by 100 undergraduate female students in a preliminary study) were chosen to compose a set of 69 unpleasant $(\mathrm{U})$ and another one of 69 neutral $(\mathrm{P})$ pictures. Both sets included the same number of pictures depicting animals, objects, faces and human beings. Unpleasant and neutral pictures differed both for valence (means $U=2.15$; $\mathrm{N}=5.17 ; \mathrm{F}(1,137)=1075.3 ; P<0.001)$ and arousal $($ means $\mathrm{U}=6.4 ; \mathrm{N}=2.8 ;(\mathrm{F}(1,137)=1280.1 ; P<0.001)$ ratings.

The mean luminance and colour saturation (for red, green and blue) for each picture were measured and some pictures were adjusted to maintain homogeneity between unpleasant and neutral sets (Adobe Photoshop ${ }^{\circledR}$ software, see $[25,34])$. Separate analyses of variance (ANOVA) performed on the mean luminance, the colour saturation and the standard deviation of luminance values (i.e. a contrast index) did not reveal any significant difference between the unpleasant and the neutral pictures (all Fs $(1,137)<2$ and $P s>0.15)$. For the spatial frequency content of the pictures, the complete procedure described by Delplanque et al. [27] was used. For each picture, the energy in spatial frequencies lower than $0.6 \mathrm{cpd}$ and higher than $4.8 \mathrm{cpd}$ was calculated and analysed for each type of layer (Red, Green, and Blue). The choice of these boundaries was based on preceding studies showing differential brain activity in function of the spatial frequency content of stimuli [35-37]. Then, data were Z-score transformed for inter-band comparability. The ANOVA performed on these scores did not reveal any significant difference between unpleasant and neutral pictures (all $\mathrm{Fs}(1,137)<2.65$ and $P$ s $>0.1)$.

\footnotetext{
${ }^{1}$ List of the IAPS identification numbers for selected pictures. Unpleasant pictures: 1052, 1090, 1111, 1114, 1201, 1932, 2053, 2120 , 2352.2, 2683, 2691, 2692, 2710, 2800, 3015, 3030, 3051, 3060, 3064, $3100,3102,3130,3150,3180,3230,3400,5940,6190,6211,6230$, $6242,6243,6312,6510,6560,6821,8480,8485,9040,9042,9160$, 9181, 9250, 9253, 9300, 9301, 9340, 9373, 9405, 9410, 9433, 9571, 9600, 9621, 9622, 9635.1, 9800, 9911, 9920, 9921. Neutral pictures: 1333, 1670, 2190, 2191, 2210, 2214, 2215, 2235, 2383, 2393, 2435, 2493, 2579, 2745.1, 2749, 2840, 2850, 2870, 2880, 2980, 5520, 5530, 7036, 7038, 7090, 7100, 7140, 7150, 7175, 7190, 7224, 7491, 7493, 7500, 7503, 7550, 7705, 7950, 8311, 9210.
} 
The whole dataset was split into three blocks of 46 pictures (23 U and $23 \mathrm{~N}$ ). For each block, all the aforementioned parameters corresponded to those of the global selection. Pictures $\left(800 \times 600\right.$ pixels; $20^{\circ}$ of visual angle at a distance of $2.1 \mathrm{~m}$ ) were randomly projected for $200 \mathrm{~ms}$ on a panoramic screen (as described in [12]), at three different eccentricities: $0^{\circ}$ (centre); $-30^{\circ}$ (left); $+30^{\circ}$ (right). Since the size of the stimuli was $20^{\circ}$, the pictures in peripheral vision extended from 20 to $40^{\circ}$ of eccentricity. The inter-stimulus interval varied between 2.5 and $4 \mathrm{~s}$. The three blocks of pictures were used for each subject. The blocks were associated with the eccentricities in such a way that, every three participants all the pictures were presented to all eccentricities.

Participants gave an informed consent, seated in a chair in front of the panoramic screen in a dimly lighted room and had to keep their eyes on a central fixation cross, their head being maintained with a chin strap. As soon as pictures appeared, they had to explicitly categorize them (i.e. unpleasant or neutral) with the help of the two buttons of a response box.

Electroencephalographic activity (EEG, ANT system) was recorded at 63 electrode sites of the extended 10-20 system using tin electrodes inserted in an elastic nylon cap (Electrocap International) with an online averaged reference and a forehead ground. Seven additional electrodes were placed for electro-ocular activity recordings: one at the nasion, two at the outer canthi of eyes and one above and below each eye. The impedance for all the electrode sites was kept below $5 \mathrm{k} \Omega$. The EEG was digitized at $1024 \mathrm{~Hz}$ in continuous. The high-low bandpass was set between 0.016 and $30 \mathrm{~Hz}$. All trials with artifacts exceeding $\pm 100 \mu \mathrm{V}$ were excluded from the analysis. Moreover, all trials containing eye movements within the first $250 \mathrm{~ms}$ after the presentation of the stimulus were rejected in order to exclude the possible saccades towards the location of visual stimulus [38]. Average-referenced EEG epochs ( -100 to $600 \mathrm{~ms}$ ) were time-locked to the stimulus onset, baseline corrected $(-100$ to $0 \mathrm{~ms})$ and averaged offline according to the a priori pictures selection.

The spatio-temporal PCA consists in two PCA on EEG waveforms to reduce both the spatial and temporal dimensionalities of the dataset. The details of the method are reported in Spencer et al. [39]. In a first step, a spatial PCA was performed, for each eccentricity, with electrode sites (63) as variables and time points, participants and conditions as observations. Spatial PCA identified groups of electrodes which were highly correlated and reduced them by hypothetical, unobserved and latent dependent variables (see [28]). Each new extracted factor can be considered as one specific spatial configuration of brain activation, called by Spencer et al. [39] "virtual electrode". The virtual electrode can be visualized by a topographic map of factor loadings, which correspond to the contribution of the virtual electrode to the original voltage at each electrode.

In a second step, the factor scores of these "virtual electrodes" (i.e. the contribution of a spatial factor to the voltage observed for each original waveform) were considered as "virtual ERPs". The corresponding time series (617 points) were submitted to a temporal PCA with time points as variables and participants and conditions as observations. Temporal PCA identifies groups of time points which are highly correlated and reduced them by hypothetical, unobserved and latent variables (i.e. temporal factors). The result of complete spatio-temporal PCA procedure is a set of factor scores used here to compare the activity of "virtual electrodes" at particular times (i.e. for each temporal factor) in response to unpleasant and neutral pictures presented at each visual eccentricity.

\section{Results}

\section{Behavioural Performance}

A Greenhouse-Geisser corrected repeated measures ANOVA with eccentricity $\left(-30^{\circ}, 0^{\circ},+30^{\circ}\right)$ and emotional content (neutral, unpleasant) as within-subject factors was performed on the rate of correct categorization responses. This analysis revealed a significant interaction between eccentricity and emotional content $(\mathrm{F}(2,34)=15.91$; $P<0.001$ ). Post hoc comparisons (Tuckey's HSD) revealed a higher rate of correct responses for neutral pictures than for unpleasant ones, when they were presented in the left $\left(-30^{\circ}: \mathrm{N}=89.2 \% \pm 10.8 ; \mathrm{U}=\right.$ $53.7 \% \pm 19.6 ; P<0.001)$ and in the right visual field $\left(+30^{\circ}: \quad \mathrm{N}=90.6 \% \pm 11.1 ; \quad \mathrm{U}=51.5 \% \pm 18.1 ; \quad P<\right.$ $0.001)$ whereas there was no significant difference for central vision $(\mathrm{N}=95.1 \% \pm 10.4 ; \mathrm{U}=85.7 \% \pm 14.5$; $P=0.21$ ). Moreover, post hoc comparisons show that percentages of correct responses for neutral pictures were not different across spatial positions $\left(-30^{\circ}\right.$ vs. $0^{\circ}$ : $P=0.612 ;-30^{\circ}$ vs. $+30^{\circ}: P=0.987 ; 0^{\circ}$ vs. $+30^{\circ}$ : $P=0.934)$. Finally, percentages of correct responses for unpleasant pictures did not differ from chance level when they were presented in the left $(\mathrm{t}=0.84 ; P=0.588)$ or in the right $(\mathrm{t}=0.65 ; P=0.474)$ visual field.

A similar ANOVA was performed on the participant's mean reaction time, according to the a priori selection of the pictures. This analysis revealed a main effect of eccentricity (quadratic trend: $\mathrm{F}(1,17)=19.34 ; P<0.001$ ) corresponding to longer reaction times in response to the peripherally presented pictures as compared to the centrally presented ones $\left(-30^{\circ}=981 \pm 155 \mathrm{~ms} ; \quad 0^{\circ}=891 \pm\right.$ $\left.132 \mathrm{~ms} ;+30^{\circ}=984 \pm 168 \mathrm{~ms}\right)$. Moreover, a main effect of emotional content was also significant $(\mathrm{F}(1,17)=4.44$; 
$P<0.05)$ with longer reaction times in response to unpleasant pictures $(\mathrm{U}=969 \pm 136 \mathrm{~ms} ; \quad \mathrm{N}=933 \pm$ $154 \mathrm{~ms})$. The interaction between emotional content and eccentricity did not reach significance $(F(1,17)=0.85)$.

\section{Eccentricity Effects on Early ERPs}

In order to investigate the early occipital activity (P100) in response to both centrally and peripherally presented pictures (Fig. 1a), we first conducted a baseline-to-peak analysis (seeking for maximum amplitude and latency) in a 70-110 ms temporal window after stimulus onset for three occipital electrodes ( $\mathrm{Oz}$ for the midline, PO7 and PO8 for left and right hemispheres, respectively). A GreenhouseGeisser corrected repeated measures ANOVA was performed with emotional content (unpleasant, neutral) eccentricity $\left(-30^{\circ}, 0^{\circ},+30^{\circ}\right)$ and electrode (PO7, Oz, PO8) as within-subject factors on the measured amplitudes. This analysis revealed a significant eccentricity $\mathrm{x}$ electrodes interaction $(\mathrm{F}(4,68)=8.99 ; \quad P<0.001)$ allowing the investigation of each eccentricity separately. When the pictures were presented to the left $\left(-30^{\circ}\right)$, the amplitude significantly increased from the left to the right parietooccipital areas (linear trend: $\mathrm{F}(1,17)=19.67 ; P<0.001$ ). For the centrally presented pictures $\left(0^{\circ}\right)$, the amplitude was higher for the midline electrode $(\mathrm{Oz})$ as compared to the lateral ones (PO7 and PO8; quadratic trend: $\mathrm{F}(1,17)=5.18 ; P<0.05)$, the amplitude at the left side being slightly inferior to the right side (PO7 < PO8; linear trend: $\mathrm{F}(1,17)=5.82 ; P<0.05)$. When the pictures were presented to the right $\left(+30^{\circ}\right)$, the amplitude significantly increased from the right to the left parieto-occipital areas (linear trend: $\mathrm{F}(1,17)=6.82 ; P<0.05$ ) (Fig. 1b). This analysis did not reveal any significant emotional effect or significant interaction between spatial position and emotional content of the pictures.

We also investigated potential peak latency differences by performing a Greenhouse-Geisser corrected repeated measures ANOVA with eccentricity $\left(-30^{\circ}, 0^{\circ},+30^{\circ}\right)$ and electrode (PO7, Oz, PO8) as within-subject factors. A main effect of eccentricity (quadratic trend: $\mathrm{F}(1,17)=8.78$; $P<0.01)$ revealed longer latencies in response to peripherally presented pictures $\left(-30^{\circ}=99 \pm 9 \mathrm{~ms} ; 0^{\circ}=95 \pm\right.$ $9 \mathrm{~ms} ;+30^{\circ}=102 \pm 8 \mathrm{~ms}$ ).
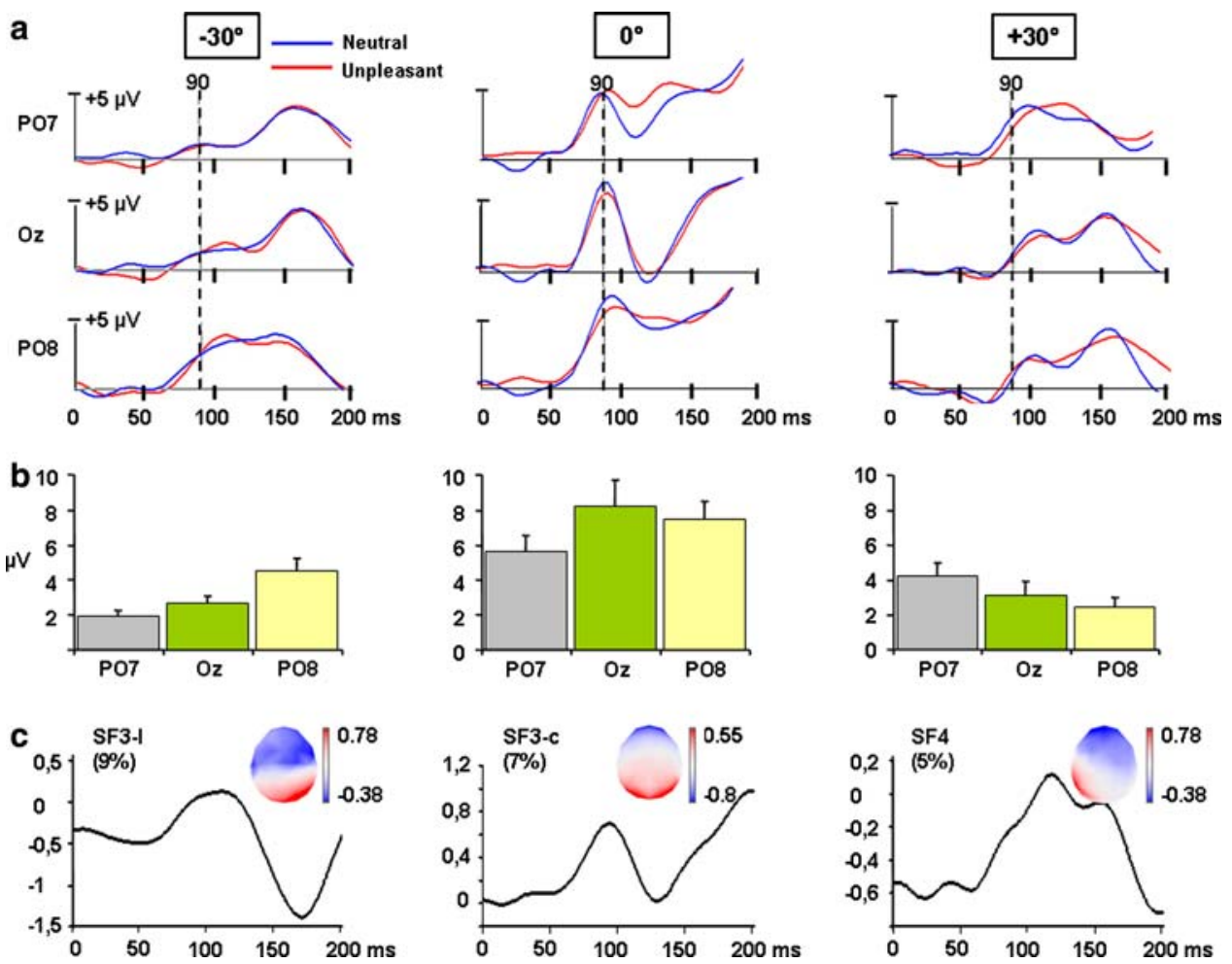

Fig. 1 Effects of stimulus location (either central or peripheral) on early ERPs. (a) Averaged ERPs elicited in response to neutral (N, blue line) and unpleasant ( $\mathrm{U}$, red line) pictures presented at three eccentricities $\left(-30^{\circ}, 0^{\circ},+30^{\circ}\right)$ and recorded at left $(\mathrm{PO} 7)$, median $(\mathrm{Oz})$ and right (PO8) parieto-occipital electrodes. (b) Mean peak voltages calculated between 70 and $110 \mathrm{~ms}$ (peak at $90 \mathrm{~ms}$, broken vertical line in (a) of the early occipital activity (P100) for the same eccentricities and electrodes. (c) Grand average spatial factor scores (virtual ERPs) across conditions ( $\mathrm{U}$ and $\mathrm{N}$ ) for spatial factors. The value of the factor scores (y axis) is a unitless dimension. Topographic maps of the factor loadings for the spatial factors (virtual electrodes) and the percentage of total variance explained by these factors is indicated 
Among the "virtual electrodes" (spatial factors: SF) extracted by the spatial PCAs performed for each eccentricity, three topographies (SF3-1, for the third SF extracted by PCA applied to data of $-30^{\circ}$ presentation; SF3-c, for the third SF extracted by PCA applied to $0^{\circ}$ presentation; $\mathrm{SF} 4$, at $+30^{\circ}$ ) showed an increase of activity around $100 \mathrm{~ms}$ after the onset of the stimulus, as showed by the representation of their "virtual ERPs" (Fig. 1c), and could be associated with the classical P100 component. In sum, when pictures were presented in left visual field $\left(-30^{\circ}\right)$, a P100-like with an increase of activity around 100-120 ms was observed in contralateral side of presentation, i.e. in right parieto-occipital areas (SF3-1). Conversely, when pictures were presented in right visual field $\left(+30^{\circ}\right)$, a P100like corresponding to an increase of activity around 100$120 \mathrm{~ms}$, was observed in left parieto-occipital areas (SF4). Finally, the centrally presented pictures $\left(0^{\circ}\right)$ induced an increase of activity in a broad and mainly median occipital area $(\mathrm{SF} 3-\mathrm{c})$ at around $80-100 \mathrm{~ms}$ that can be reasonably associated with the P100.

\section{Emotional Effects on Early ERPs}

For each couple composed by a spatial and a temporal factor (i.e. the activity of a "virtual electrode" at a specific time), an ANOVA with emotional content (U, N) as within-subject factor was performed on the individual temporal factor

scores. For better readability, we represented (Fig. 2) only the comparisons that demonstrated significant differences for the emotional content during the early visual activity after the stimulus onset $(<250 \mathrm{~ms})$. When the pictures were presented to the left $\left(-30^{\circ}\right.$, Fig. $\left.2 \mathrm{a}\right)$, the first significant effect of emotional content appears for the P100-like, after about $145 \mathrm{~ms}$ (as indicated by the maximum of TF6 loadings) mainly on right parieto-occipital areas (as indicated by the maximum of SF3-1 loadings) with higher scores in response to neutral pictures as compared to unpleasant ones $(\mathrm{F}(1,17)=6.15 ; P<0.05)$. Later on $(225 \mathrm{~ms}$; TF4), neutral pictures elicited less activity than unpleasant ones mainly on frontal areas $(\mathrm{SF} 1 ; \mathrm{F}(1,17)=9.4 ; P<0.01)$ but also on right temporal areas $(\mathrm{SF} 5 ; \mathrm{F}(1,17)=13.39 ; P<0.01)$. When pictures were presented in the centre (Fig. 2b), the first emotional effect was not observed for the P100 (SF3-c) but appeared slightly before, around $90 \mathrm{~ms}$ (TF8) with higher factor scores for neutral pictures than for unpleasant pictures on parietal areas $(\mathrm{SF} 2 ; \mathrm{F}(1,17)=6.33 ; P<0.05)$. Later differences were observed (145 ms, TF7) for the same areas (SF2) with higher activity for neutral pictures than for unpleasant ones $(\mathrm{F}(1,17)=4.53 ; P<0.01)$. Finally, when the pictures were presented to the right $\left(+30^{\circ}\right.$, Fig. $\left.2 \mathrm{c}\right)$, the activity recorded for the P100-like at about $123 \mathrm{~ms}$ (TF7) was significantly lower for neutral pictures as compared to unpleasant ones on left parieto-occipital areas (SF4; $\mathrm{F}(1,17)=6.92 ; P<0.05)$. Later differences were observed

b
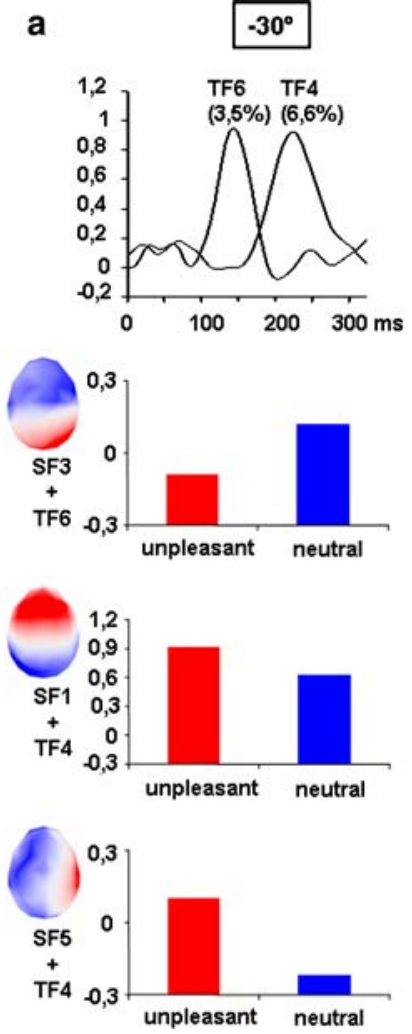

c
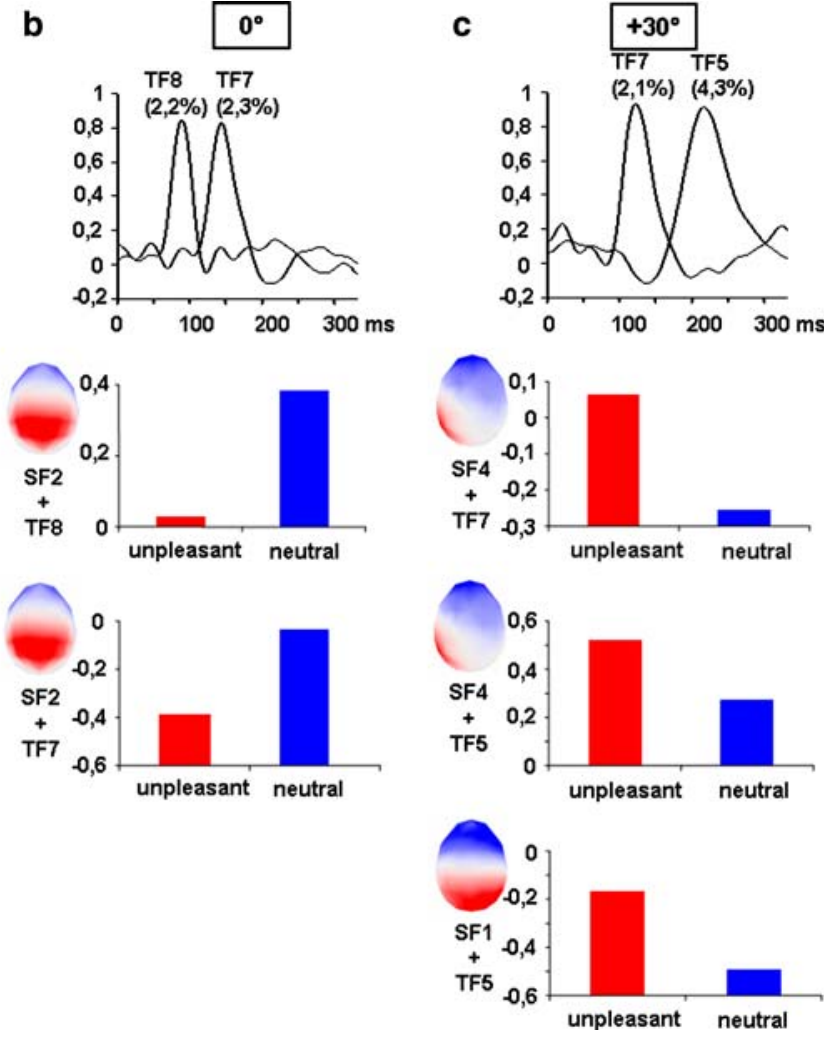
at about $220 \mathrm{~ms}$ (TF5) with lower activity in response to neutral pictures as compared to unpleasant ones for left parieto-occipital areas $(\mathrm{SF} 4 ; \mathrm{F}(1,17)=9.4 ; P<0.01)$ and also for frontal areas $(\mathrm{SF} 1 ; \mathrm{F}(1,17)=6.2 ; P<0.05)$.

\section{Discussion}

The objective of the current study was to examine the early visual evoked responses to complex pictures presented at peripheral locations and to assess the existence of early emotional processing in those conditions.

\section{Eccentricity and Emotional Effects on Behavior}

First, the global performance of the participants decreased with eccentricity since the percentage of correct responses of participants significantly dropped. Moreover, the decrease of correct responses in peripheral vision was more pronounced for unpleasant pictures. One could argue that this result is due to a bias in our a priori selection of pictures, the neutral ones being more easily categorized. However, since the participants categorized correctly both neutral and unpleasant pictures when presented in central vision, such a proposal is highly improbable. In our opinion, this result is better explained by a response bias, most of the participants reporting that they used the neutral choice when they were not sure about the pictures content. This strategy artificially increased the ratio of correct responses for neutral pictures, which remained high across the different eccentricities. By contrast, the percentage of correct response for unpleasant pictures decreased to chance level when presented at the periphery.

Second, the reaction times were longer in response to pictures projected in peripheral visual fields as compared to centrally presented ones, irrespective of their emotional content. These results, in line with those reported by Thorpe et al. [13] for the presentation of non emotional natural scenes, can be explained by a decrease of global vision quality in periphery. As suggested by these authors, the reaction times are thought to increase with the spacing between retinal ganglion cells, observed from fovea to periphery. Moreover, the reaction times were globally longer in response to unpleasant pictures, whatever the eccentricity. The unpleasant content of the pictures could have captured more attentional resources than the neutral ones, thus reducing resources available for the categorization task and then explaining the increase in reaction times for these pictures. Indeed, several studies support this assumption [40-42] by showing that target detection at invalid locations was slowed by threat cues, suggesting that unpleasant stimuli could engage more attentional resources at the emotionally cued location.
Finally, considering that the carefully designed selection of a priori unpleasant and neutral pictures matched the participant's categorization in central vision, the electrophysiological analyses were focused on same pictures selection.

\section{Effects of Eccentricity on Early Visual ERPs}

When pictures were presented in far peripheral vision, the baseline-to-peak analysis as well as the spatio-temporal PCA revealed the existence of an electrophysiological component similar to the P100 (called here P100-like) already described in central vision $[43,44]$. The $\mathrm{P} 100$ reflects early activation in extrastriate visual areas, mainly in lateral occipital and inferior temporal cortices [45]. This origin could explain the topographical distribution observed for both central and peripheral presentations in our study. Indeed, in peripheral vision, this "P100-like" component is lower in amplitude, slightly delayed and its distribution is dependent on the side of presentation of the visual stimulus. As a matter of fact, whereas the P100 evoked by centrally presented pictures was maximally distributed in occipital median electrode, the "P100-like" evoked by peripherally presented pictures was maximal for the parieto-occipital areas contralateral to the stimulus presentation side. Indeed, visual information coming from the right visual hemifield is conveyed to the left occipital visual areas (and vice-versa for the left visual hemifield) via both ipsilateral and contralateral pathways. Such predominant contralateral activity in response to peripherally presented pictures was already found with fMRI [46], magnetoencephalography [47] or visual evoked potentials [48] but never at such eccentric locations. The lateralization of the early visual activity could also be related to a more extended and direct innervation of secondary and more lateral areas by the magnocellular pathway, as suggested by a few studies in animals ([49] in monkeys; [50] in cats). Our study constitutes a first insight into the early visual processing of complex pictures presented at high eccentricity. However, more psychophysical studies are needed to refine the neural network involved in far eccentricity processing, as it was done in parafoveal vision (i.e. [51]).

\section{Effects of Emotion on Early Visual ERPs}

The third and main result of our study is that, for the first time, we evidenced the existence of early emotional processing at far peripheral vision. This result confirms that the peripheral vision could process stimuli having a very high adaptive value and that emotional content can be extracted even in peripheral vision, i.e. in conditions of low acuity. It extends results of Calvo and Lang's study which showed emotional differentiation in parafoveal retina $\left(10^{\circ}\right.$ of eccentricity). 
Early emotional differences are observed for both central and peripheral vision and are unlikely related to the perceptual characteristics of the stimulus since luminance, contrast and spatial frequency content of the pictures were controlled. In central vision, the first modulation due to the emotional content of the picture was observed earlier than $100 \mathrm{~ms}$ on parieto-occipital areas. This kind of effect was already described in this latency range but rarely on the peak of the P100 itself $[15,52]$. In far peripheral vision $\left(+30^{\circ}\right.$ and $\left.-30^{\circ}\right)$, the earliest emotional influence was observed in the contralateral occipital area, after $120 \mathrm{~ms}$, i.e. after the aforementioned P100-like component and after the earliest observed emotional effects for central presentations. This latency difference could be due to the difficulty to extract the emotional features of the scenes presented to high eccentricities. These early emotional differences could be explained by top-down feedback, from higher cortical structures to visual areas [53], or by modulation in the sensory visual areas themselves. There is also evidence that the amygdala processes aversive information very rapidly $[54,55]$. Given its connections with the visual cortex, amygdala [53] could rapidly process unpleasant stimulus and contribute to dissociate neutral from unpleasant information in extrastriate areas. Later stages of stimulus processing were also modulated by the emotional content when pictures were presented both in central and peripheral vision. Indeed, spatial and temporal factors showed differential sensitivity to emotional content according to eccentricity which suggests the existence of different neural networks involved in emotional processing for central and peripheral vision.

Our analyses revealed an early emotional effect with neutral pictures eliciting stronger activities than unpleasant ones, when presented in the left and the central visual fields. This unexpected result could be considered as rather inconsistent with the classic ERP pattern showing enhanced ERPs effects for unpleasant stimuli compared to neutral ones [19]. However, other studies showed higher early [56] or late [57] ERP amplitudes for neutral compared to unpleasant stimuli, at least in central vision. Thus, the classical view of amplitudes superiority for emotional laden stimuli should not be considered as a systematic rule. Moreover, concerning peripheral presentations, to our knowledge, there is no available data on ERPs emotional effects. In sum, the complex pattern of results obtained by exploratory spatiotemporal analysis asks for further studies in order to better identify emotional effects elicited in paradigms conjugating central and peripheral vision exploration.

In addition, considering the higher temporal resolution of the magnocellular pathway, mostly related to the peripheral vision [4], it seems counter-intuitive that emotional effects appeared earlier in central than in peripheral vision. However, recent data [58] showed that magnocellular and parvocellular pathways are not clearly separated and that many connections exist between them. Thus, it seems premature to conclude that the magnocellular pathways alone would convey the emotional related information. Future studies using moving or low-passed filtered stimuli rather than static pictures containing all the spatial frequencies, could be of great help to shed some light on this issue.

In conclusion, the ability of central vision to process emotional information appeared to persist in peripheral vision, in spite of structural differences. This supports the view that affective content is of the greatest importance for adaptation, whatever conditions of vision. In line with the studies of masking, rapid serial visual presentations, or using unexpected emotional stimuli, this work strongly suggests that human brain is able to quickly disentangle affective from neutral information in low acuity vision.

Acknowledgements This study was supported by a grant of the Ministère de la Recherche of France to S.R. and funds from CNRS and Université de Lille I to H.S. Authors are grateful to Dr J. Polich for helpful comments on the electrophysiological data.

\section{References}

1. Rousselet GA, Thorpe SJ, Fabre-Thorpe M. How parallel is visual processing in the ventral pathway? Trends Cogn Sci 2004;8:363-70.

2. Liversedge SP, Findlay JM. Saccadic eye movements and cognition. Trends Cogn Sci 2000;4:6-14.

3. Dacey DM, Petersen MR. Dendritic field size and morphology of midget and parasol ganglion cells of the human retina. Proc Natl Acad Sci USA 1992;89:9666-70.

4. Livingstone MS, Hubel DH. Psychophysical evidence for separate channels for the perception of form, color, movement, and depth. J Neurosci 1987;7:3416-68.

5. Schiller PH, Logothetis NK, Charles ER. Functions of the colouropponent and broad-band channels of the visual system. Nature 1990;343:68-70.

6. Chung STL, Mansfield JS, Legge GE. Psychophysics of reading. XVIII. The effect of print size on reading speed in normal peripheral vision. Vision Res 1998;38:2949-62.

7. Nasanen R, O'Leary C. Recognition of band-pass filtered handwritten numerals in foveal and peripheral vision. Vision Res 1998;38:3691-701.

8. Anderson RS. Aliasing in peripheral vision for counterphase gratings. J Opt Soc Am A Opt Image Sci Vis 1996;13:2288-93.

9. Jüttner M, Rentschler I. Scale-invariant superiority of foveal vision in perceptual categorization. Eur J Neurosci 2000;12:353-9.

10. Loftus GR, Mackworth NH. Cognitive determinants of fixation location during picture viewing. J Exp Psychol Hum Percept Perform 1978;4:565-72.

11. Nelson WW, Loftus GR. The functional visual field during picture viewing. J Exp Psychol Human Percept Perform 1980;6: 391-9.

12. Nailli F, Despretz P, Boucart M. Colour recognition at large visual eccentricities in normal observers and patients with low vision. Neuroreport 2006;17:1571-4.

13. Thorpe SJ, Gegenfurtner K, Fabre-Thorpe M, Bülthoff HH. Detection of animals in natural images using far peripheral vision. Eur J Neurosci 2001;14:869-76. 
14. Lang PJ, Bradley MM, Cuthbert BN. Motivated attention: affect, activation, action. In: Lang PJ, Simons RF, Balaban MT, editors. Attention and orienting: sensory and motivational processes. Hillsdale, NJ: Erblaum; 1997. pp. 97-135.

15. Smith NK, Cacioppo JT, Larsen JT, Chartrand TL. May I have your attention, please: electrocortical responses to positive and negative stimuli. Neuropsychologia 2003;41:171-83.

16. Buchanan TW. Retrieval of emotional memories. Psychol Bull 2007;133:761-79.

17. Labar KS, Cabeza R. Cognitive neuroscience of emotional memory. Nat Rev Neurosci 2006;7:54-64.

18. Phelps EA. Human emotion and memory: interactions of the amygdala and hippocampal complex. Curr Opin Neurobiol 2004;14:198-202.

19. Schupp HT, Junghöfer M, Weike AI, Hamm AO. Emotional facilitation of sensory processing in the visual cortex. Psychol Sci 2003;14(1):7-13.

20. Delplanque S, Silvert L, Hot P, Rigoulot S, Sequeira H. Arousal and valence effects on event-related P3a and P3b during emotional categorization. Int J Psychophysiol 2006;60:315-22.

21. Carretié L, Hinojosa JA, Mercado F, Tapia M. Cortical response to subjectively unconscious danger. Neuroimage 2005;24:615-23.

22. Junghöfer M, Bradley MM, Elbert TR, Lang PJ. Fleeting images: a new look at early emotion discrimination. Psychophysiology 2001;38:175-8.

23. Silvert L, Delplanque S, Bouwalerh H, Verpoort C, Sequeira H. Autonomic responding to aversive words without conscious valence discrimination. Int J Psychophysiol 2004;53:135-45.

24. Williams MA, Morris AP, McGlone F, Abbott DF, Mattingley JB. Amygdala responses to fearful and happy facial expressions under conditions of binocular suppression. $\mathbf{J}$ Neurosci 2004;24:2898-904.

25. Calvo MG, Lang PJ. Parafoveal semantic processing of emotional visual scenes. J Exp Psychol Hum Percept Perform 2005;31:502_ 19.

26. Lang PJ, Bradley MM, Cuthbert BN (2001) International affective picture system (IAPS): instruction manual and affective ratings. Technical report A-5, The center for research in Psychophysiology, University of Florida.

27. Delplanque S, N'Diaye K, Scherer K, Grandjean D. Spatial frequencies or emotional effects? A systematic measure of spatial frequencies for IAPS pictures by a discrete wavelet analysis. J Neurosci Methods 2007;165:144-50.

28. Pourtois G, Delplanque S, Michel C, Vuilleumier P. Beyond conventional event-related brain potential (ERP): exploring the time-course of visual emotion processing using topographic and principal component analyses (this issue).

29. Carretié L, Iglesias J, Barry RJ. Parietal N300 elicited by emotional visual stimulation. J Psychophysiol 1998;12:376-83.

30. Delplanque S, Lavoie ME, Hot P, Silvert L, Sequeira H. Modulation of cognitive processing by emotional valence studied through event-related potentials in humans. Neurosci Lett 2004;356:1-4.

31. Hot P, Saito Y, Mandai O, Kobayashi T, Sequeira H. An ERP investigation of emotional processing in European and Japanese individuals. Brain Res 2006;1122:171-8.

32. Kayser J, Tenke CE. Optimizing PCA methodology for ERP component identification and measurement: theoretical rationale and empirical evaluation. Clin Neurophysiol 2003;114:2307-25.

33. Hécaen H. Les gauchers: Etude Neurophysiologique. Paris: PUF; 1984.

34. Ochsner KN. Are affective events richly recollected or simply familiar? The experience and process of recognizing feelings past. J Exp Psychol Gen 2000;129:242-61.

35. Kenemans JL, Baas JMP, Mangun GR, Lijffijt M, Verbaten MN. On the processing of spatial frequencies as revealed by evokedpotential source modeling. Clin Neurophysiol 2000;111:1113-23.
36. Baas JMP, Kenemans JL, Mangun GR. Selective attention to spatial frequency: an ERP and source localization analysis. Clin Neurophysiol 2002;113:1840-54.

37. Winston JS, Vuilleumier P, Dolan RJ. Effects of low-spatial frequency components of fearful faces on fusiform cortex activity. Curr Biol 2003;13:1824-9.

38. Rayner K. Eye movements in reading and information processing: 20 years of research. Psychol Bull 1998;124:372-422.

39. Spencer KM, Dien J, Donchin E. Spatiotemporal analysis of the late ERP responses to deviant stimuli. Psychophysiology 2001;38:343-58.

40. Fox E, Russo R, Bowles R, Dutton K. Do threatening stimuli draw or hold visual attention in subclinical anxiety? J Exp Psychol Gen 2001;130:681-700.

41. Koster EHW, Crombez G, Van Damme S, Verschuere B, De Houwer J. Does imminent threat capture and hold attention? Emotion 2004;4:312-7.

42. Mathews A, Yiend J, Lawrence AD. Individual differences in the modulation of fear-related brain activation by attentional control. J Cogn Neurosci 2004;16:1683-94.

43. Hopf JM, Mangun GR. Shifting visual attention in space: an electrophysiological analysis using high spatial resolution mapping. Clin Neurophysiol 2000;111:1241-57.

44. Clark VP, Hillyard SA. Spatial selective attention affects early extrastriate but not striate components of the visual evoked potential. J Cogn Neurosci 1996;8:387-402.

45. Pourtois G, Grandjean D, Sander D, Vuilleumier P. Electrophysiological correlates of rapid spatial orienting towards fearful faces. Cereb Cortex 2004;14:619-33.

46. Whittingstall K, Stroink G, Schmidt M. Evaluating the spatial relationship of event-related potential and functional MRI sources in the primary visual cortex. Hum Brain Mapp 2007;28:134-42.

47. Liu L, Ioannides AA. Spatiotemporal dynamics and connectivity pattern differences between centrally and peripherally presented faces. Neuroimage 2006;15:1726-40.

48. Fize D, Fabre-Thorpe M, Richard G, Doyon B, Thorpe SJ. Rapid categorization of foveal and extrafoveal natural images: associated ERPs and effects of lateralization. Brain Cogn 2005;59:145-58.

49. Foster KH, Gaska JP, Nagler M, Pollen DA. Spatial and temporal frequency selectivity of neurones in visual cortical areas v1 and v2 of the macaque monkey. J Physiol 1985;365:331-63.

50. Movshon JA, Thompson ID, Tolhurst DJ. Spatial and temporal contrast sensitivity of neurones in areas 17 and 18 of the cat's visual cortex. J Physiol 1978;283:101-20.

51. Clark VP, Fan S, Hillyard SA. Identification of early visual evoked potential generators by retinotopic and topographical analyses. Human Brain Mapping 1995;2:170-87.

52. Carretié L, Hinojosa JA, Martin-Loeches M, Mercado F, Tapia M. Automatic attention to emotional stimuli: neural correlates. Hum Brain Mapp 2004;22:290-9.

53. Amaral DG, Behniea H, Kelly JL. Topographic organization of projections from the amygdala to the visual cortex in the macaque monkey. Neuroscience 2003;118:1099-120.

54. Morris JS, Öhman A, Dolan RJ. Conscious and unconscious emotional learning in the human amygdala. Nature 1998; 393:467-70.

55. Davidson RJ, Irwin W. The functional neuroanatomy of emotion and affective style. Trends Cogn Sci 1999;3:11-21.

56. Begleiter H, Gross MM, Kissin B. Evoked cortical responses to affective visual stimuli. Psychophysiology 1967;3:336-44.

57. Kayser J, Tenke CE, Nordby H, Hammerborg D, Hugdah K, Erdmann G. Event-related potential (ERP) asymmetries to emotional stimuli in a visual half-field paradigm. Psychophysiology 1997:34:414-26.

58. Sincich LC, Horton JC. The circuitry of V1 and V2: integration of color, form and motion. Annu Rev Neurosci 2005;28:303-26. 